\title{
LAPLACE TRANSFORM PAIRS OF N-DIMENSIONS
}

\section{R. S. DAHIYA}

Department of Mathematics

Iowa State University

Ames, Iowa 50011

(Received February 22, 1982)

ABSTRACT. In this paper I prove a theorem to obtain new n-dimensional Laplace transform pairs.

KEY WORDS AND PHRASES. Laplace transforms, multiple transforms. 1980 AMS SUBJECT CIASSIFICATION CODE. $44 \mathrm{~A} 30$

1. INTRODUCTION.

The generalization of the well-known Laplace transform

$$
L\{f(t) ; s\}=\int_{0}^{\infty} \exp (-s t) f(t) d t
$$

to n-dimensional Laplace transform is represented as follows:

$$
\begin{aligned}
& L_{n}\left\{f\left(t_{1}, t_{2}, \ldots t_{n} ; s_{1}, s_{2}, \ldots, s_{n}\right\}=L_{n}\{f\}\right. \\
= & \int_{0}^{\infty} \int_{0}^{\infty} \cdots \int_{0}^{\infty} \exp \left(-\sum_{k=1}^{n} s_{k} t_{k}\right) f t_{1} d t_{2} \ldots d t_{n} \cdot(1.2)
\end{aligned}
$$

In this paper I consider a method of computing Laplace transform pairs of ndimensions from known one-dimensional Laplace transforms. The multi-dimensional Laplace transform pairs are useful in the solution of partial differential equations (see [1], [3] and [4]).

2. THEOREM. Let

$$
\begin{aligned}
& \text { (i) } \mathrm{L}_{1}\{\mathrm{f}(\mathrm{t}) ; \mathrm{s}\}=\phi(\mathrm{s}) \\
& \text { (ii) } \mathrm{L}_{1}\left\{\sqrt{\mathrm{t}} \phi\left(\frac{1}{\mathrm{t}}\right) ; \mathrm{s}\right\}=\mathrm{F}(\mathrm{s}) \\
& \text { (iii) } \mathrm{L}_{1}\left\{\mathrm{t}^{3} \mathrm{f}\left(\mathrm{t}^{4}\right) ; \mathrm{s}\right\}=\mathrm{G}(\mathrm{s}) \\
& \text { (iv) } \mathrm{L}_{1}\left\{\mathrm{t}^{4} \mathrm{f}\left(\mathrm{t}^{4}\right) ; \mathrm{s}\right\}=\mathrm{H}(\mathrm{s})
\end{aligned}
$$


and let $f(t), \sqrt{t} \phi\left(\frac{1}{t}\right), t^{3} f\left(t^{4}\right), t^{4} f\left(t^{4}\right)$ be continuous and absolutely integrable in $(0, \infty)$. Then

$$
\begin{aligned}
& L_{n}\left\{\frac{\left(\frac{1}{t_{1}}+\ldots+\frac{1}{t_{n}}\right)^{3}}{\left(t_{1} \ldots t_{n}\right)^{1 / 2}} F\left[\frac{1}{64}\left(\frac{1}{t_{1}}+\ldots+\frac{1}{t_{n}}\right)^{2}\right] ; s_{1}, \ldots, s_{n}\right\} \\
& =2^{10} \pi^{\frac{n+1}{2}} \frac{G\left(\bar{s}_{1}+\ldots+\sqrt{s_{n}}\right)}{\left(s_{1} \ldots s_{n}\right)^{1 / 2}}+2^{9} \pi^{\frac{n+1}{2}} \frac{\sqrt{s_{1}}+\ldots+\sqrt{s_{n}}}{\left(s_{1} \ldots s_{n}\right)^{1 / 2}} H\left(\sqrt{s_{1}}+\ldots+\sqrt{s}_{n}\right), \\
& n=2,3,4 \ldots
\end{aligned}
$$

provided the integral on the left exists as an absolutely convergent in each of the variables.

PROOF: From (i), we have

$$
\begin{gathered}
\phi\left(\frac{1}{s}\right)=\int_{0}^{\infty} e^{-t / s} f(t) d t=\int_{0}^{\infty} e^{-u / s} f(u) d u, \\
\sqrt{t} \phi\left(\frac{1}{t}\right)=\int_{0}^{\infty} \sqrt{t} e^{-u / t} f(u) d u .
\end{gathered}
$$

Let us multiply both sides of $(2.2)$ by $e^{-s t}, \operatorname{Re}(s)>0$, and integrate between the limits $(0, \infty)$. Then on changing the order of integrations on the resulting right hand integral (permissible by Fubini's theorem, on account of absolute convergence), we obtain

$$
\int_{0}^{\infty} e^{-s t} \sqrt{t} \phi\left(\frac{1}{t}\right) d t=\int_{0}^{\infty} f(u)\left[\int_{0}^{\infty} \sqrt{t} e^{-s t-u / t} d t\right] d u .
$$

We then evaluate the inner integral on the right (see [5], page 22) and use (ii) on the left to get the following result:

$$
\begin{aligned}
& F(s)=\frac{\sqrt{\pi}}{2} \int_{0}^{\infty}(1+2 \sqrt{u s}) s^{-3 / 2} e^{-2 \sqrt{u s}} f(u) d u, \\
& s^{3 / 2} F(s)=\frac{\sqrt{\pi}}{2} \int_{0}^{\infty}(1+2 \sqrt{u s}) e^{-2 \sqrt{u s}} f(u) d u .
\end{aligned}
$$

Next let us write $(2.3)$ in the form

$$
\begin{aligned}
& \left(\frac{1}{t_{1}}+\ldots+\frac{1}{t_{n}}\right)^{3} F\left[\frac{1}{64}\left(\frac{1}{t_{1}}+\ldots+\frac{1}{t_{n}}\right)^{2}\right] \\
& =256 \sqrt{\pi} \int_{0}^{\infty} e^{-\frac{\sqrt{u}}{4} \sum \frac{1}{t_{i}}} f(u) d u+64 \sqrt{\pi} \int_{0}^{\infty}\left(\frac{1}{t_{1}}+\ldots+\frac{1}{t_{n}}\right) e^{-\frac{\sqrt{u}}{4} \sum \frac{1}{t_{i}}} \cdot \sqrt{u} f(u) d u
\end{aligned}
$$

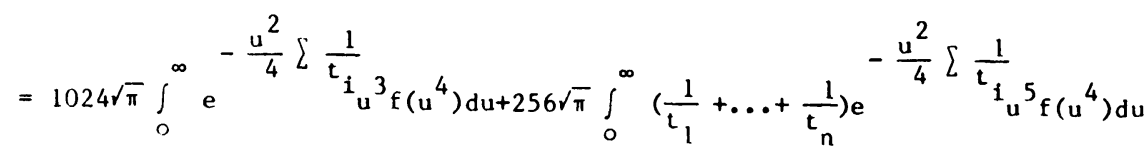


We multiply both sides by $\left(t_{1} \ldots t_{n}\right)^{-1 / 2} \exp \left(-\sum s_{i} t_{i}\right)$, integrate with respect to $t_{1}$ between the limits $(0, \infty)$ and then change the order of integrations in the resulting integral on the right, permissible by Fubini's theorem, on account of absolute convergence.

This gives

$$
\begin{aligned}
& \int_{0}^{\infty} \cdots \int_{0}^{\infty} \exp \left(-\sum s_{i} t_{i}\right) \frac{\left(\frac{1}{t_{1}}+\ldots+\frac{1}{t_{n}}\right)^{3}}{\left(t_{1} \ldots t_{n}\right)^{1 / 2}} F\left[\frac{1}{64}\left(\frac{1}{t_{1}}+\ldots+\frac{1}{t_{n}}\right)^{2}\right] d t 1 \ldots d t_{n} \\
& =1024 \sqrt{\pi} \int_{0}^{\infty} u^{3} f\left(u^{4}\right)\left[\int_{0}^{\infty} \frac{1}{\sqrt{t}} \exp \left(-s_{1} t_{1}-\frac{u^{2}}{4 t_{1}}\right) d t_{1} \ldots \int_{0}^{\infty} \frac{1}{\sqrt{t_{n}}} \exp \left(-s_{n} t_{n}-\frac{u^{2}}{4 t}\right) d t n\right] d u \\
& +256 \sqrt{\pi} \int_{0}^{\infty} u^{5} f\left(u^{4}\right)\left[\int_{0}^{\infty} \ldots \int_{0}^{\infty}\left(\frac{1}{t_{1}^{3 / 2} \sqrt{t_{2} \cdots t_{n}}}+\frac{1}{\sqrt{t_{1}} t_{2}^{3 / 2} \sqrt{t_{3} \cdots t_{n}}}+\ldots+\frac{1}{\sqrt{t_{1} \cdots t_{n-1} t_{n}^{3 / 2}}}\right)\right. \\
& \left.\exp \left(-s_{i} t_{i}-\frac{u^{2}}{4} \sum \frac{1}{t_{i}}\right) d t_{1} \ldots d_{n}\right] d u
\end{aligned}
$$

Evaluating the inner integrals on the right by (see [5], page 22, results 6 and 7)

$\int_{0}^{\infty} \frac{1}{\sqrt{t}} \exp \left(-s t-\frac{u^{2}}{4 t}\right) d t=\sqrt{\frac{\pi}{s}} e^{-u \sqrt{s}}, \int_{0}^{\infty} t^{-3 / 2} \exp \left(-s t-\frac{u^{2}}{4}\right) d t=\frac{2 \sqrt{\pi}}{u} e^{-u \sqrt{s}}$

we get

$$
\begin{aligned}
& L_{n}\left\{\frac{\left(\frac{1}{t_{1}}+\ldots+\frac{1}{t_{n}}\right)^{3}}{\left(t_{1} \ldots t_{n}\right)^{1 / 2}} F\left[\frac{1}{64}\left(\frac{1}{t_{1}}+\ldots+\frac{1}{t_{n}}\right)^{2}\right] ; s_{1}, \ldots, s_{n}\right\} \\
& =1024 \pi^{\frac{n+1}{2}}\left(s_{1} \ldots s_{n}\right)^{-1 / 2} \int_{0}^{\infty} \exp \left(-u\left[\sqrt{s_{i}}\right) u^{3} f\left(u^{4}\right) d u\right. \\
& +512 \pi^{\frac{n+1}{2}} \frac{\left(\sqrt{s_{1}}+\ldots+\sqrt{s_{n}}\right)}{\left(s_{1} \ldots s_{n}\right)^{1 / 2}} \int_{0}^{\infty} \exp \left(-u\left[\sqrt{s_{i}}\right) u^{4} f\left(u^{4}\right) d u\right.
\end{aligned}
$$

The proof is complete if we use (iii) and ((iv) on the right hand side of (2.5).

3. APPLICATIONS: n-dimensional Laplace transform pairs.

Let $\mathrm{f}(\mathrm{t})=\mathrm{t}^{\mathrm{v}}$; so that $\mathrm{L}_{1}\left\{\mathrm{t}^{\mathrm{v}} ; \mathrm{s}\right\}=\frac{\Gamma(\mathrm{v}+1)}{\mathrm{s}^{\mathrm{v}+1}}=\phi(\mathrm{s})$. Then $\mathrm{L}_{1}\left\{\sqrt{\mathrm{t}} \phi\left(\left(\frac{1}{\mathrm{t}}\right), \mathrm{s}\right\}=\mathrm{L}_{1}\left\{\Gamma(\mathrm{v}+1) \mathrm{t}^{\mathrm{v}+3 / 2} ; \mathrm{s}\right\}=\frac{\Gamma(\mathrm{v}+1) \Gamma(\mathrm{v}+5 / 2)}{\mathrm{x}^{\mathrm{v}+5 / 2}}=\mathrm{F}(\mathrm{s})\right.$, 


$$
\begin{aligned}
& L_{1}\left\{t^{3} f\left(t^{4}\right) ; s\right\}=L_{1}\left\{t^{4 v+3} ; s\right\}=\frac{\Gamma(4 v+4)}{s^{4 v+4}}=G(s) \\
& \begin{aligned}
L_{1}\left\{t^{4} f\left(t^{4}\right) ; s\right\}=L_{1}\left\{t^{4 v+4} ; s\right\}=\frac{\Gamma(4 v+t)}{s^{4 v+5}}=H(s) \text {. Hence from }(2.1) \text {, we get } \\
L_{n}\left\{\left(t_{1} \ldots t_{n}\right)^{-1 / 2}\left(\frac{1}{t_{1}}+\ldots+\frac{1}{t_{n}}\right)^{-2 v-2} ; s_{1}, \ldots, s_{n}\right\} \\
=\frac{\pi^{\frac{n+1}{2}} \Gamma(4 v+4)}{8^{2 v-7} \Gamma(v+1) \Gamma(v+5 / 2)}\left(s_{1} \ldots s_{n}\right)^{-1 / 2}\left(\sqrt{s_{1}}+\ldots+\sqrt{s_{n}}\right)^{-4 v-4} \\
\quad+\frac{\pi^{\frac{n+1}{2}} \Gamma(4 v+5)}{8^{2 v-6} \Gamma(v+1) \Gamma(v+5 / 2)}\left(s_{1} \ldots s_{n}\right)^{-1 / 2}\left(\sqrt{s_{1}}+\ldots+\sqrt{s_{n}}\right)^{-4 v-4} .(3.1)
\end{aligned}
\end{aligned}
$$

Similarly if we take $f$ to be the following

$$
f(t)=\left\{\begin{array}{l}
t^{c-1} \circ^{F_{3}(a, b, c ; k t)} \\
t^{v} \exp (-\sqrt{t}) \\
J_{v}^{2}(\sqrt{2 t} \\
t^{\alpha}{ }_{p} F_{q}\left(\begin{array}{l}
(a) ; t \\
(b) ; t
\end{array}\right)
\end{array}\right.
$$

in the theorem, then we obtain the following n-dimensional Laplace transform pairs:

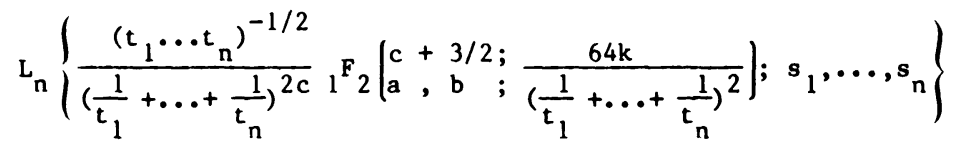

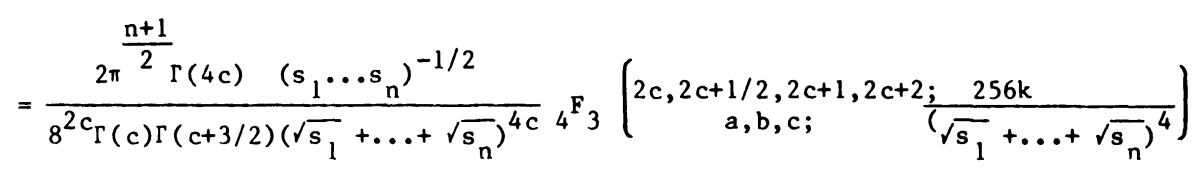

$$
\begin{aligned}
& +\frac{\pi^{\frac{n+1}{2}} \Gamma(4 c+1)\left(s_{1} \ldots . s_{n}\right)^{-1 / 2}}{8^{2 c} \Gamma(c) \Gamma(c+3 / 2)\left(\sqrt{s_{1}}+\ldots+\sqrt{s_{n}}\right)^{4 c}}{ }_{4} F_{3}\left(\begin{array}{c}
2 c+1 / 2,2 c+1,2 c+3 / 2,2 c+2 ; \\
a, b, c ;
\end{array} \frac{256 k}{\left(\sqrt{s_{1}}+\ldots+\sqrt{s_{n}}\right)^{4}}\right), \\
& \operatorname{Re}(c)>0 \text {. }
\end{aligned}
$$




$$
\begin{aligned}
& L_{n}\left\{\frac{\frac{1}{t_{1}}+\ldots+\frac{1}{t_{n}}}{\left(t_{1} \ldots t_{n}\right)^{1 / 2}}-\left(\frac{1}{64}\left(\frac{1}{t_{1}}+\ldots+\frac{1}{t_{n}}\right)^{2}-\frac{1}{4}\right)^{-v-3 / 2} p_{1}^{-2 v-3}\left(\frac{4}{\frac{1}{t_{1}}+\ldots+\frac{1}{t_{n}}}\right) ; s_{1}, \ldots, s_{n}\right\} \\
& =\frac{\pi^{n / 2} \Gamma(4 v+4)}{2^{v-4} \Gamma(2 v-5)}\left(s_{1} \ldots s_{n}\right)^{-1 / 2} \exp \left(\frac{1}{8}\left(\sqrt{s_{1}}+\ldots+\sqrt{s_{n}}\right)^{2}\right) D_{-4 v-4}\left(\frac{1}{\sqrt{2}}\left(\sqrt{s_{1}}+\ldots+\sqrt{s_{n}}\right)\right)
\end{aligned}
$$

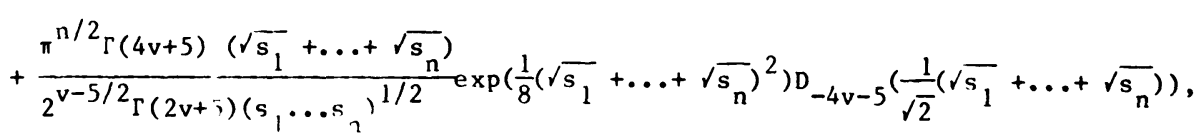

$$
\begin{aligned}
& L_{n}\left\{\frac{\left(t_{1} \ldots n_{n}\right)^{-1 / 2}\left(\frac{1}{t_{1}}+\ldots+\frac{1}{t_{n}}\right)^{3}}{\left(\frac{1}{64}\left(\frac{1}{t_{1}}+\ldots+\frac{1}{t_{n}}\right)^{2}+1\right)-1} Q_{v-1 / 2}^{2}\left(\frac{1}{64}\left(\frac{1}{t_{1}}+\ldots+\frac{1}{t_{n}}\right)^{2}+1\right) ; s_{1}, \ldots, s_{n}\right\} \\
& =\frac{\pi^{\frac{n}{2}+1} \sin (v+3 / 2) \pi\left(\sqrt{s_{1}}+\ldots+\sqrt{s_{n}}\right)^{v / 2}}{2^{\frac{v}{8}-9} \sin (v-1 / 2) \pi\left(s_{1} \ldots s_{n}\right) 1 / 2} \\
& \text { - } G_{50}^{03}\left(\frac{8 \sqrt{2}}{\left(\sqrt{s_{1}}+\ldots+\sqrt{s_{n}}\right)^{2}} \mid 2-\frac{v}{3},-\frac{v}{4},-\frac{1}{2}-\frac{v}{4}, \frac{3}{2}+\frac{v}{4}, 2+\frac{v}{4}\right) \\
& +\frac{\pi^{\frac{n}{2}+1} \sin (v+3 / 2) \pi\left(\sqrt{s_{1}}+\ldots+{\sqrt{s_{n}}}^{\frac{v+3}{2}}\right.}{s^{v-73 / 8} \sin (v-1 / 2) \pi\left(s_{1} \ldots s_{n}\right)^{1 / 2}} . \\
& G_{50}^{03}\left(\frac{8 \sqrt{2}}{\left(\sqrt{s_{1}}+\ldots+\sqrt{s_{n}}\right)^{2}} \mid \frac{9}{4}-\frac{v}{4},-\frac{1}{4}-\frac{v}{4},-\frac{3}{4}-\frac{v}{4}, \frac{7}{4}+\frac{v}{4}, \frac{9}{4}+\frac{4}{4}\right) \\
& L_{n}\left\{\frac{\left(t_{1} \ldots t_{n}\right)^{-1 / 2}}{\left(\frac{1}{t_{1}}+\ldots+\frac{1}{t_{n}}\right)^{2 \alpha+2} p+2^{F}}\left(\frac{(a), \alpha+1, \alpha+5 / 2 ;}{(b) ;} \frac{64}{\left(\frac{1}{t_{1}}+\ldots+\frac{1}{t_{n}}\right)^{2}}\right) ; s_{1}, \ldots, s_{n}\right\}
\end{aligned}
$$$$
=\frac{\pi^{\frac{n+1}{2}} \Gamma(4 \alpha+4)\left(s_{1} \ldots s_{n}\right)^{-1 / 2}}{4(8)^{2 \alpha+1} \Gamma(\alpha+1) \Gamma(\alpha+5 / 2)\left(\sqrt{s_{1}}+\ldots+\sqrt{s_{n}}\right)^{4 \alpha+4}}
$$$$
\text { - } p+4 F_{q}\left(\begin{array}{l}
(a), 2 \alpha+2,2 \alpha+5 / 2,2 \alpha+3,2 \alpha+7 / 2 ; \\
\text { (b); }
\end{array}\right.
$$$$
+\frac{\pi^{\frac{n+1}{2}} \Gamma(4 \alpha+5)\left(s_{1} \ldots s_{n}\right)^{-1 / 2}}{8^{2 \alpha+2} \Gamma(\alpha+1) \Gamma(\alpha+5 / 2)\left(\sqrt{s_{1}}+\ldots+\sqrt{s_{n}}\right)^{4 \alpha+4}}
$$$$
\text { - }{ }_{p+4} F_{q}\left(\begin{array}{l}
(a), 2 \alpha+3,2 \alpha+7 / 2,2 \alpha+4,2 \alpha+9 / 2 ; \\
(b) ;
\end{array}\right.
$$ 


\section{REFERENCES}

[1] Ditkin, V.A., and Prudnikov, A. P., Operational calculus in two variables and its application (English translation Pergmon Press. London (1962).

[2] Erdelyi, A. W. Magnus, F. Oberhettinger, F. Tricomi, Tables of Integral Transforms. Vol.1, McGraw-Hil1, New York (1954).

[3] Estrin, T. A. and Higgins, T. J., Solutions of boundary value problem by multiple Laplace transformation. Jour. Frank. Inst. 252 (1951), 152-167.

[4] Jaeger, J., The solution of boundary value problems by a double Laplace transformation. Bull. Amer. Math. Soc. 46 (1940), 687-693.

[5] Roberts, G. E. and Kaufman, H., Table of Laplace transforms. W. B. Saunders Company (1966). 


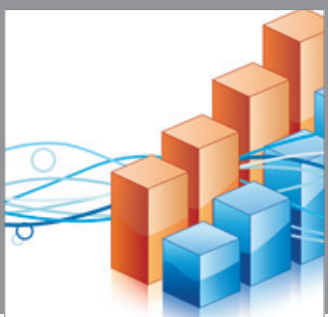

Advances in

Operations Research

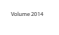

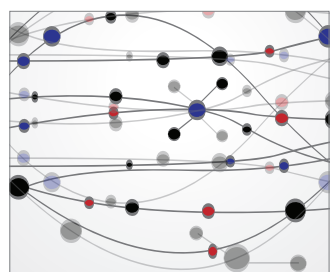

\section{The Scientific} World Journal
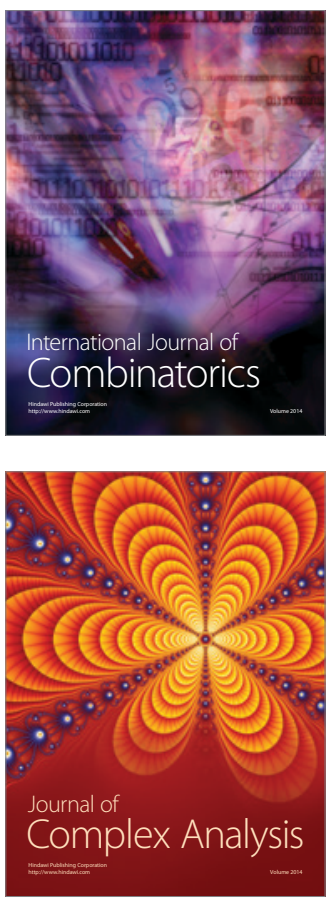

International Journal of

Mathematics and

Mathematical

Sciences
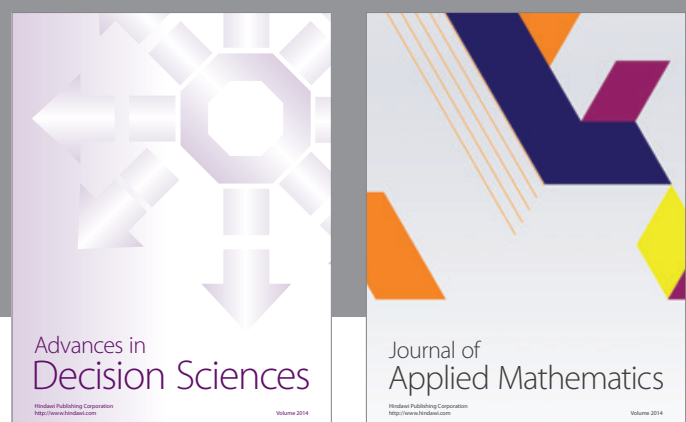

Journal of

Applied Mathematics
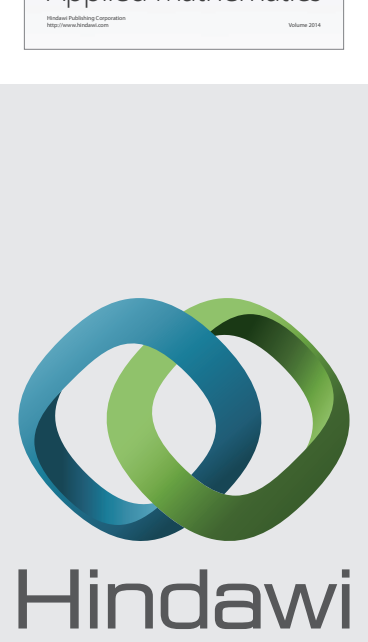

Submit your manuscripts at http://www.hindawi.com
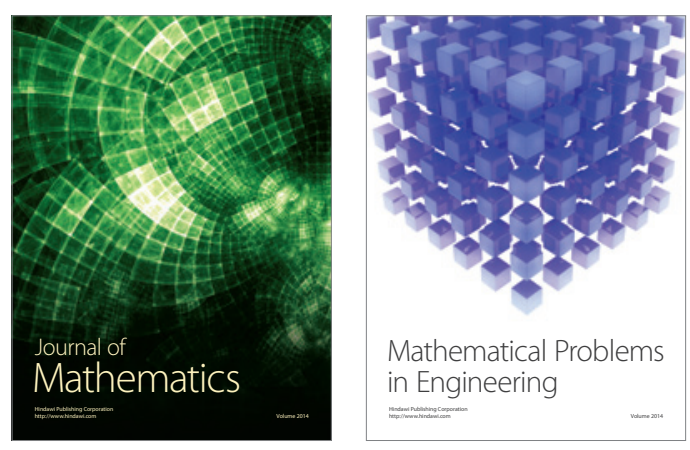

Mathematical Problems in Engineering
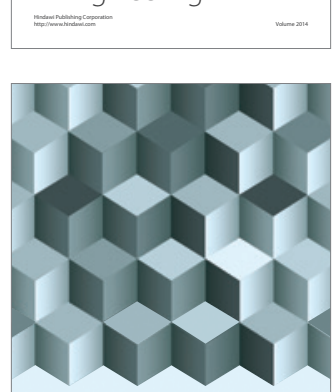

Journal of

Function Spaces
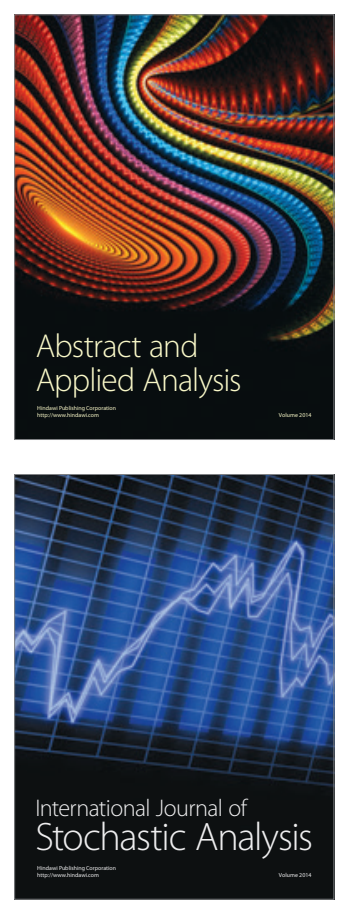

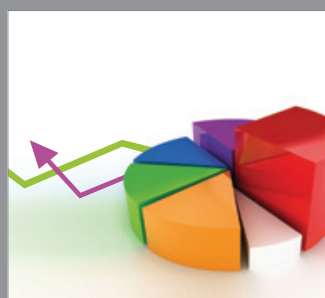

ournal of

Probability and Statistics

Promensencen
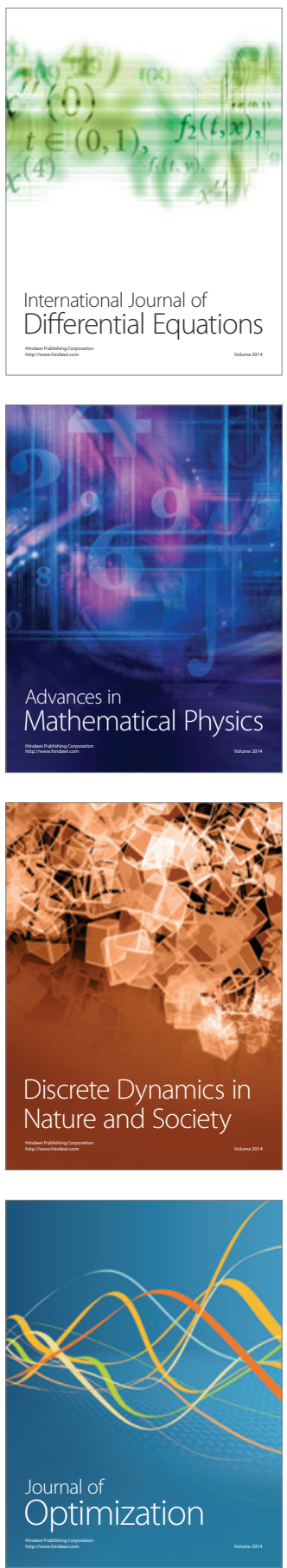